\title{
Lacrimal System Disorder
}

National Cancer Institute

\section{Source}

National Cancer Institute. Lacrimal System Disorder. NCI Thesaurus. Code C26809.

A non-neoplastic or neoplastic disorder that affects the lacrimal apparatus. 